the binding buffer, a standard and essential control. Moreover, an Argonaute with the two valine for phenylalanine substitutions showed no detectable cap binding. It seems that approximately $5 \%$ of the wild-type Argonaute input was retained on the m7GTP-Sepharose, but without comparison to a control capbinding protein, such as eIF4E, it is hard to know whether this represents moderate or even low levels of binding. Finally, Kiriakidou et al. used tagged immunopurified Argonaute from transfected 293T cells for their studies; thus, it is possible that another mammalian protein not present in Drosophila S2 cells might have aided Argonaute's interaction with m7GTP-Sepharose.
Given the results of this study by Eulalio et al., it seems that more consideration should be given to GW182 as a key factor regulating the translational activities of miRNA. The observation that GW182 promotes mRNA deadenylation and destruction ${ }^{16,17}$ obviously complicates analysis of translation; nonetheless, as long as the mechanism(s) by which miRNAs control gene expression remain(s) controversial (Fig. 1), it is essential that new approaches with additional reagents be brought to bear on this issue of overriding importance.

1. Filipowicz, W., Bhattacharyya, S.N. \& Sonenberg, N. Nat. Rev. Genet. 9, 102-114 (2008).

2. Eulalio, A., Huntzinger, E. \& Izaurralde, E. Nat. Struct. Mol. Biol. 15, 346-353 (2008).
3. Petersen, C.P. et al. Mol. Cell 21, 533-542 (2006).

4. Nottrott, S., Simard, M.J. \& Richter, J.D. Nat. Struct. Mol. Biol. 13, 1108-1114 (2006).

5. Lytle, J.R., Yario, T.A. \& Steitz, J.A. Proc. Natl. Acad. Sci. USA 104, 9667-9672 (2007).

6. Pillai, R.S. et al. Science 309, 1573-1576 (2005).

7. Humphreys, D.T. et al. Proc. Natl. Acad. Sci. USA 102 16961-16966 (2005)

8. Wakiyama, M. et al. Genes Dev. 21, 1857-1862 (2007).

9. Mathonnet, G. et al. Science 317, 1764-1767 (2007).

10. Thermann, R. \& Hentze, M.W. Nature 447, 875-878 (2007).

11. Chendrimada, T.P. et al. Nature 447, 823-828 (2007).

12. Basu, U. et al. Mol. Cell. Biol. 21, 1453-1462 (2001).

13. Ceci, M. et al. Nature 426, 579-584 (2003).

14. Kiriakidou, M. et al. Cell 129, 1141-1151 (2007).

15. Wu, L., Fan, J. \& Belasco, J.G. Proc. Natl. Acad. Sci. USA 103, 4034-4039 (2006).

16. Rehwinkel, J. et al. RNA 11, 1640-1647 (2005)

17. Behm-Ansmant, I. et al. Genes Dev. 20, 1885-1898 (2006).

\title{
No SIRT6
}

The Sir2 family of $\mathrm{NAD}^{+}$-dependent deacetylases has been associated with lifespan and chromatin silencing in yeast. Indeed, the SIRT6 mouse mutant has a decreased lifespan. The SIRT6 protein, like some other Sir2 family members, has been localized to chromatin, and new data from Chua and colleagues now indicate that it is involved in regulation of telomeric DNA during $S$ phase and suggest that the enzymatic target of SIRT6 is histone $\mathrm{H} 3$ lysine 9 (H3K9; Nature, advance online publication, 12 March 2008, doi:10.1038/ nature06736). To elucidate which processes SIRT6 might participate in, the authors virally transduced short hairpin RNAs (shRNAs) to stably knock down SIRT6 in WI-38 primary human fibroblasts. The resulting cells showed senescence after a shorter number of replicative doublings in comparison to control cells. In addition, they had increased levels of senescence markers, including damage-response markers such as $\gamma$-H2AX foci at telomeres (lower panel, $\gamma-\mathrm{H} 2 \mathrm{AX}$ in red, telomeres in green, arrowheads indicate co-localized signals) compared to control nuclei (upper panel). Moreover, increased chromosome end-to-end fusions were observed after several cell doublings, indicating defective telomeres. Although decreased telomeric sequence signals were observed in fluorescence in situ hybridization in the SIRT6 knockdown cells, global telomeric erosion was not seen. The senescence phenotype was reversed by overexpressing TERT telomerase but not DNA polymerase $\beta$. Thus, the telomeric phenotype was due to a novel telomeric function for SIRT6, rather than a previously characterized role in base excision repair.
These phenotypes resemble those of WRN, a DNA helicase defective in Werner syndrome, a condition characterized by a premature aging phenotype. The authors thus used properties of WRN to gain further insight into SIRT6 function. Like WRN, SIRT6 can be detected at telomeres using a telomeric chromatin immunoprecipitation technique. This association was most strongly observed in S-phase cells. The SIRT6 knockdown decreased WRN telomeric presence, but the reciprocal experiment revealed that SIRT6 localizes independently of WRN. This suggests that SIRT6 activity may alter telomeres in a manner required for WRN to access and modulate these chromosomal regions during $\mathrm{S}$ phase. The authors thus tested what the activity of SIRT6 might be by examining its ability to deacetylate peptides in vitro and found a modest $\mathrm{NAD}^{+}$-dependent activity specific to acetylated histone H3K9. This activity was dependent on a catalytic residue in SIRT6. Although it was difficult to detect an alteration in global histone H3K9ac levels in the SIRT6 knockdown cells, an increase in $\mathrm{H} 3 \mathrm{~K} 9 \mathrm{ac}$ levels was observed on telomeres of cells synchronized in S phase. Altogether, this body of work suggests similarities in the molecular mechanisms by which SIRT6 and WRN may prevent premature aging-like phenotypes. These observations will allow further work elucidating the mechanism by which SIRT6 is involved in WRN localization, as well as whether and how its deacetylation activity targeting $\mathrm{H} 3 \mathrm{~K} 9 \mathrm{ac}$ is responsible for the observed telomeric phenotypes.

Sabbi Lall 